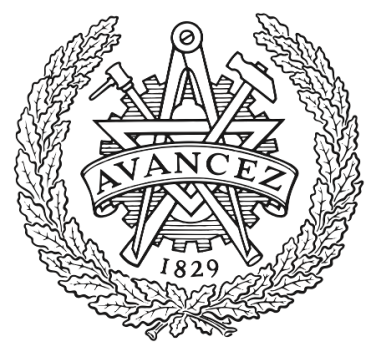

CHALMERS

UNIVERSITY OF TECHNOLOGY

\title{
High Capacity Transmission Systems Using Homogeneous Multi-Core Fibers [Invited]
}

Downloaded from: https://research.chalmers.se, 2023-04-26 12:20 UTC

Citation for the original published paper (version of record):

Puttnam, B., Luis, R., Agrell, E. et al (2017). High Capacity Transmission Systems Using Homogeneous Multi-Core Fibers [Invited]. Journal of Lightwave Technology, 35(6): 1157-1167. http://dx.doi.org/10.1109/JLT.2017.2669207

N.B. When citing this work, cite the original published paper. 


\title{
High Capacity Transmission Systems Using Homogeneous Multi-Core Fibers
}

\author{
Benjamin J. Puttnam, Member, IEEE, Ruben S. Luís Member, IEEE, Erik Agrell, Member, IEEE, \\ Georg Rademacher, Member, IEEE, Jun Sakaguchi, Member, IEEE, Werner Klaus, Member, OSA, \\ George M. Saridis, Student Member, IEEE, Yoshinari Awaji, Member, IEEE, and Naoya Wada, \\ Member, IEEE
}

\begin{abstract}
We discuss transmission using homogeneous, single-mode, multi-core fibers. We first describe the key fiber properties that can affect transmission, such as inter-core crosstalk and the variation of propagation delay between cores, and the consequences of these properties on spatial super-channel transmission. We then describe a series of experiments and demonstrations of system features including self-homodyne detection and joint spatial super-channel modulation before discussing their suitability to high-capacity transmission.
\end{abstract}

Index Terms - multi-core fibers, space-division multiplexing

\section{INTRODUCTION}

$\mathrm{O}$ ptical networks and transmission systems are a key part of the worldwide communications infrastructure, underpinning the digital economy and supporting the intensive data networking needs of industry, commerce, academic institutions, governments and individuals worldwide [1]. However, optical communication infrastructure is constantly challenged by ever-increasing traffic demands combined with commercial pressure to offer them at ever lower cost per bit. Space-division-multiplexing (SDM) technologies [2] and networking [3] have been widely proposed as cost-effective solutions with multiple fiber cores [4] and various spatial-modes [5], [6] being used to increase the number of transmission channels in a single fiber. Indeed, few-mode (FM) multi-core fibers (MCFs) with over 100 spatial channels have now been reported [7], [8] and single fiber capacity exceeding $2 \mathrm{~Pb} / \mathrm{s}$ demonstrated [9], [10].

Homogeneous, single-mode (SM)-MCFs perhaps offer the simplest migration path for adoption of high-capacity SDM technology in the near term. Such fibers have been shown to support high spectral efficiency (SE) modulation formats and wide band operation without the complexity of high-order multiple input-multiple output (MIMO) based receivers [10]. Homogeneous SM-MCFs have been used in long-haul transmission [11], access [12], data-centre [13] and networking demonstrations [14]. Also, SM-MCFs may be fabricated with

B. J. Puttnam, R. S. Luis, G. Rademacher, J. Sakaguchi, W. Klaus, Y. Awaji and N. Wada are members of the Photonic Network System Laboratory, National Institute of Information and Communications Technology (NICT), 4-2-1 Nukui-Kitamachi, Koganei, Tokyo 184-8795, Japan. (e-mail: ben@nict.go.jp).

E. Agrell is with the Department of Signals and Systems, Chalmers University of Technology, SE-412 96 Gothenburg, Sweden.

G.M. Saridis is with High Performance Networks Group, University of Bristol, United Kingdom. relatively low crosstalk (XT) and a smaller cladding diameter than equivalent core number FM-MCFs, making splicing and handling easier. Furthermore, the relative uniformity of the homogeneous cores support spatial super channels (SSCs) for shared transmitter hardware, digital signal processing (DSP) and simplified switching [15]. Additional system advantages can exploit the similar propagation characteristics of different SDM channels pilot-tone aided transmission schemes [16], [17] or multi-dimensional modulation and coding [18]. In this paper, we expand on results presented in [19], and describe recent progress on high-capacity systems using homogeneous SM-MCFs, the key fiber properties and the potential of system features to further increase efficiency in high-capacity SDM transmission.

\section{Properties Of Homogeneous, Single-Mode MCFs}

\section{A. Inter-core crosstalk}

XT between spatial channels is a potential limitation of SDM systems. In the case of MCFs, the impact of inter-core (IC) XT (ICXT) is strongly dependent on the relative group velocities of signals travelling in different cores [20]. Hence for homogeneous MCFs with near identical refractive indices and propagation properties between cores, the impact of ICXT may be strong, depending on the core spacing or pitch [20]. ICXT
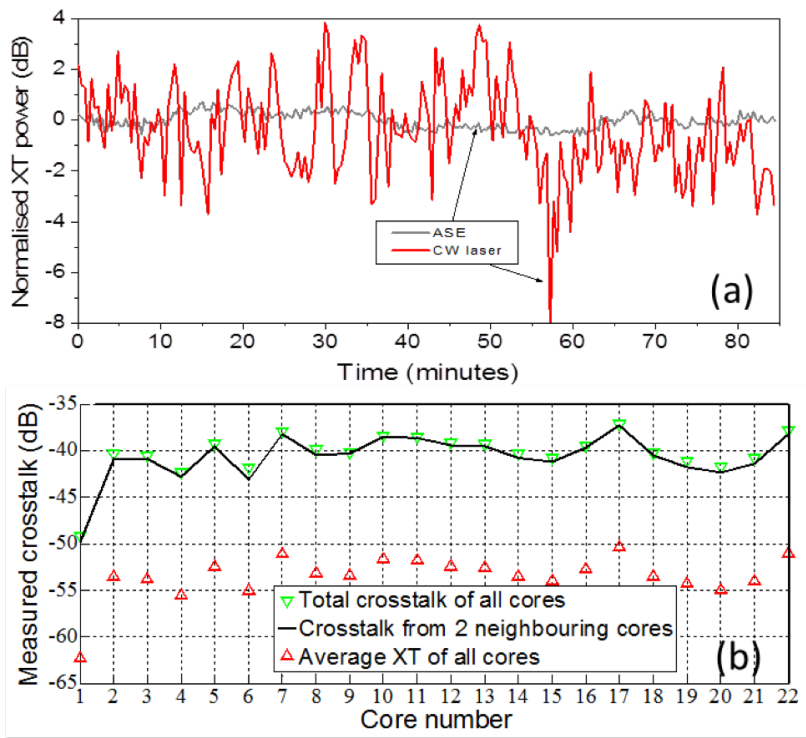

Fig. 1. (a) Normalized short term average XT from neighbouring core of a 22-core MCF from CW laser filtered ASE (b) measured average crosstalk in each of a 22-core fiber 
differs from localized crosstalk in optical components in the sense that it has a stochastic behavior, varying randomly over time and wavelength within a wide dynamic range and depending strongly on the inter-core temporal skew and signal modulation [20]-[23]. These studies have shown that ICXT results from relatively complex interference mechanisms and may affect transmission performance differently than in-band $\mathrm{XT}$ added at a single point, as investigated in [24]. Fig. 1(a) shows the time varying crosstalk from a continuous wave (CW) laser signal and from amplified spontaneous emission (ASE) noise filtered at $0.2 \mathrm{~nm}$ over a period exceeding 80 minutes. It is shown that although significant ICXT fluctuations may be observed in both cases, the wider spectral occupation of the $0.2 \mathrm{~nm}$ ASE yields substantially lower ICXT fluctuations than the $\mathrm{CW}$.

Experimental investigations of the impact of ICXT on the transmission of QPSK [24] and higher order square quadrature amplitude modulation (QAM) formats [25] showed that above a threshold of around $-40 \mathrm{~dB} / 100 \mathrm{~km}$, XT can limit the achievable transmission distance, particularly for higher order modulation formats. Furthermore, in order to guarantee a quality of service in the presence of randomly varying XT power, additional optical signal-to-noise ratio (OSNR) margin may be required, further limiting throughput [25]. Hence, various strategies to reduce IC coupling have been proposed including novel fibre designs [26], [27] and transmission schemes such as bi-directional interleaving [28]. Indeed, ICXT measurements of a 22-core fiber [10], shown in Fig. 1(b), reveal that such a fiber would suit bi-directional transmission since the 2 core pitch design means that the major XT contribution comes from neighboring cores in the same ring, allowing ICXT well below $-40 \mathrm{~dB} / 100 \mathrm{~km}$.

MCFs with a heterogeneous core design are also suitable candidates for long-haul transmission, with generally lower ICXT. Furthermore, the XT in such fibers does not increase with bending radius above a critical radius [29] and may allow higher core-count fibers without increasing the core-pitch [29]. However, recent measurements of unspooled homogeneous fibers suggest that the dependence of ICXT on bending radius is not as severe as expected with little increase when XT was compared in a straightened $2 \mathrm{~km}$ span and the same fiber spooled with $16 \mathrm{~cm}$ radius [30]. To date, the MCF with lowest ICXT reported [20] $(\approx 64.7 \mathrm{~dB} / 100 \mathrm{~km}$ at $1550 \mathrm{~nm})$ used a homogeneous core design.

\section{B. Dynamic inter-core skew variations}

For advanced SDM systems based around SSCs the arrival time of sub-channels at the receiver is an important consideration. Although framing and buffering techniques may be used to mitigate their impact, large variation of propagation delays are still likely to impact the achievable baud-rates, transmission distances, receiver design and complexity of DSP [15], [31]. This is particularly significant for transmission over many spans or in networks where paths are dynamically assigned. However, only a few studies on randomly varying propagation delay between cores, referred to here as dynamic IC-skew, in homogeneous MCFs have been reported to date

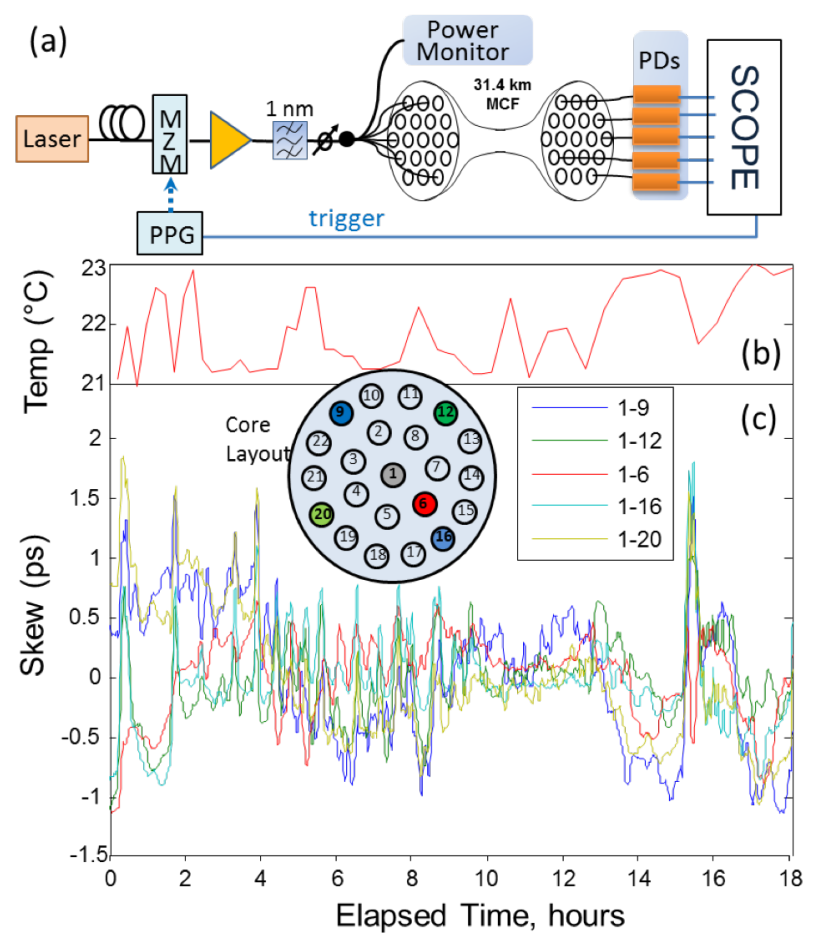

Fig. 2. (a) Experimental set-up (b) ambient temperature and (c) measured IC skew of 6 outer-cores relative to the center core (1) over 18 hours

[21], [32]. The dynamic IC-skew for core pairs of a 7-core MCF was also compared with SMF and shown to vary by almost 2 orders of magnitude less than SMF, over a 24 hour period [33].

Figure 2(a) shows the set-up for dynamic skew measurement in a 22 core fiber [10] in laboratory conditions. A Mach-Zehnder modulator (MZM) was driven by a pulse pattern generator (PPG) to generate a $10 \mathrm{~Gb} / \mathrm{s}$ on-off keying (OOK) signal using a $2^{7}-1$ pseudo random binary sequence (PRBS). This signal was amplified and split between multiple cores of an MCF. After fiber transmission, the signals were received by individual photodetectors (PDs) connected to a sampling oscilloscope synchronised with the PPG. The relative dynamic skew between pairs of received cores was then estimated by cross-correlating the corresponding signals, as in [21]. Fig. 2 (c) shows the measured dynamic skew of 5 cores of the 22 core fiber used in [10] relative to the centre cores over an 18 hour period. Fig. 2(b) shows the ambient temperature around the spooled fiber. Although the total fluctuation is only around $2^{\circ} \mathrm{C}$, measurement of several fibers suggested a correlation between of temperature fluctuations and the fine detail of the skew variation. In this measurement and similar measurements with MCFs with 7 and 19 cores [32], some sensitivity to mechanical vibrations caused by lab activity was also observed during the measurement period. Despite this, the largest measured skew variation between cores in the 22-core MCF was under $4 \mathrm{ps}$ and under 2 ps for 5 of the 6 cores measured.

\section{EXPERIMENTS WITH HOMOGENEOUS MCFS}

In the following sections, we describe various experiments investigating aspects of transmission systems based on homogeneous MCFs. Specific details of experiments are described in the corresponding sections, but features common 
to all set-ups are described in this section.

\section{A. Homogeneous Multi-Core Fibers}

Experiments in the following sections used $3 \mathrm{MCF}$ designs with 7, 19 and 22 cores. Their main properties are listed in table 1. The 7 core and 22-core fiber used 3D waveguide technology for coupling of light in and out whilst the 19-core fiber used free-space couplers.

TABLE I

PROPERTIES OF MCFS USED IN EXPERIMENTS

\begin{tabular}{|ccclclc|}
\hline $\begin{array}{l}\text { Core } \\
\text { No. }\end{array}$ & $\begin{array}{c}\text { Length } \\
(\mathbf{k m})\end{array}$ & $\begin{array}{l}\text { Average } \\
\text { loss } \\
(\mathbf{d B} / \mathbf{k m})\end{array}$ & $\begin{array}{l}\text { Core } \\
\text { pitch } \\
(\mathbf{u m})\end{array}$ & $\begin{array}{l}\text { Cladding } \\
\text { diameter } \\
\text { (um) }\end{array}$ & $\begin{array}{l}\text { Core } \\
\text { layout }\end{array}$ & Ref. \\
\hline \hline 7 & $\mathbf{2 8 . 4}$ & $\mathbf{0 . 1 8 4}$ & $\mathbf{4 4 . 2}$ & $\mathbf{1 6 0}$ & Hex. & {$[\mathbf{2 1}]$} \\
19 & $\mathbf{3 0}$ & $\mathbf{0 . 2 8 5}$ & $\mathbf{3 9} / \mathbf{3 7 . 6}$ & $\mathbf{2 2 0}$ & Hex. & {$[34]$} \\
22 & $\mathbf{3 1 . 4}$ & $\mathbf{0 . 2}$ & $\mathbf{4 2} / \mathbf{4 9}$ & $\mathbf{2 6 0}$ & Circ. & {$[10]$} \\
\hline
\end{tabular}

B. Wide-band comb

Many of the experiments described used a custom designed wide band frequency comb source, made by RAM Photonics. The large bandwidth of the comb, first used in [10], has shown to be a vital component in achieving $\mathrm{Pb} / \mathrm{s}$ class transmission with the moderate amount of spatial channels available in single-mode MCFs. It consists of a narrow linewidth $(5 \mathrm{kHz})$ seed laser at $1559 \mathrm{~nm}$ modulated with a low noise $25 \mathrm{GHz}$ oscillator to provide $25 \mathrm{GHz}$ spaced comb lines. The modulated seed laser is amplified and spectrally broadened in a dispersion-engineered fiber mixer [35], [36] to provide spectral coverage from $1510 \mathrm{~nm}$ to $1620 \mathrm{~nm}$, although it was not possible to utilize its full range due to bandwidth limitations of other components. The total output power was 29 to $30 \mathrm{dBm}$ with the power per line varying by $20 \mathrm{~dB}$ over the utilized spectral coverage due to the large peak at the comb seed, evident in Fig. 3(a). For transmission experiments, the comb output was flattened using separate $\mathrm{C}$ and L-band optical processors, as in [10], to provide a spectrum shown in Fig. 3(b) with the $1 \mathrm{~nm}$ guard band required for spectral separation shown as inset 2. Internal closed-loop controls were used to maintain stable long-term operation. However, drift in relative power of up to $3 \mathrm{~dB}$ was observed for comb lines close to the comb seed

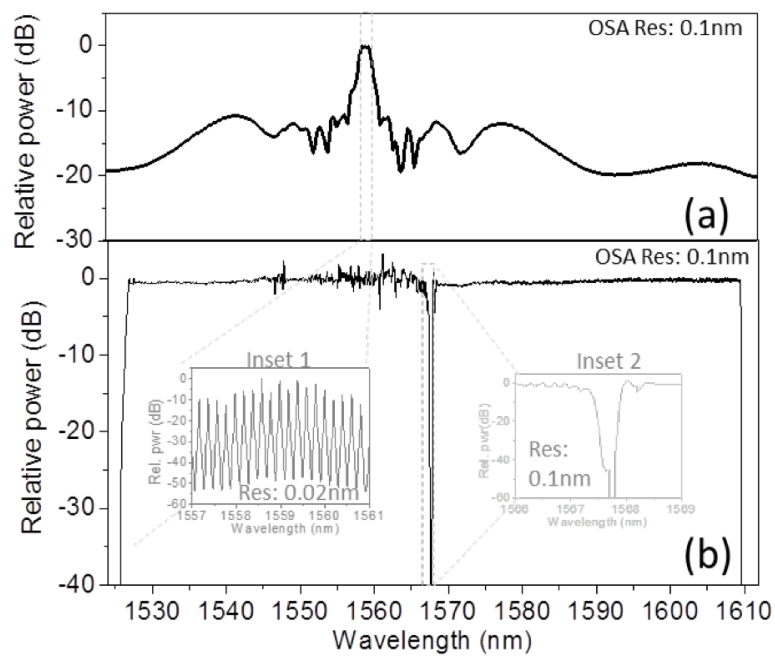

Fig. 3. Optical spectra of (a) Comb output without gain-equalization and its center band (Inset1), and (b) flattened comb output showing C and L channel guard band (Inset 2)

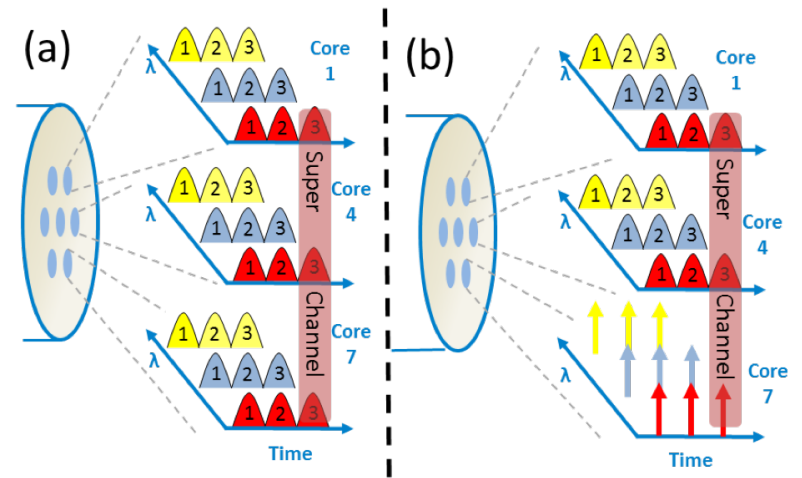

Fig. 4. (a) 3-core spatial super-channels in 3 different wavelength and (b) 2-core self-homodyne spatial superchannels in 3 wavelengths

and up to $0.5 \mathrm{~dB}$ at the comb edges. The measured OSNR was $>$ $45 \mathrm{~dB}$ at $1535 \mathrm{~nm}$ and $>47 \mathrm{~dB}$ at $1554 \mathrm{~nm}$.

\section{Signal modulation and reception}

Signal modulation was performed using a pair of dual parallel MZMs, subsequently referred to as DP-QAM modulators, driven by 4 independent arbitrary waveform generators (AWG). Each AWG had an analogue bandwidth of $14 \mathrm{GHz}$ and used a sampling rate of $49 \mathrm{GS} / \mathrm{s}$ to generate pre-equalized polarization-division-multiplexed (PDM)-QAM signals with root-raised cosine pulse shape and a roll-off factor of 0.01 . For WDM experiments using the comb source a Baud-rate of 24.5 GBaud was used but single channel experiments used variable baud rates described in each case. Using independent AWGs allowed the programming of specific core-dependent bit patterns to each quadrature and polarization to enable core-specific patterns to be implemented with the same transmitter.

Signal reception was performed in a polarization-diverse optical coherent receiver (CoRx) connected to a digital sampling oscilloscope with $32 \mathrm{GHz}$ analogue bandwidth operating at $80 \mathrm{GS} / \mathrm{s}$. Offline processing was used to recover the signals, consisting of resampling to 2 samples per symbol, followed by normalization and dispersion compensation. Polarization de-multiplexing was performed using a $2 \times 2$ MIMO structure whose equalizers were 17-tap filters updated using a decision-directed least-mean square (DD-LMS) algorithm. Carrier recovery was embedded in the equalizer loop. Bit error ratios (BER) were calculated from the average of three $10 \mu$ s traces.

\section{SPATIAL SUPER CHANNEL TRANSMISSION}

Spatial super-channels (SSCs) were proposed with the aim of sharing DSP resources common to all cores [15]. An SSC comprises signals of the same wavelength in multiple cores and may be all or a subset of MCF cores, as shown in Fig. 4(a) over 3 cores of a 7-core fiber. Hardware savings are possible by sharing a single laser between multiple cores, and SSCs have also been used to simplify switching in reconfigurable add and drop multiplexers (ROADMs) using MCF links [37], [38]. In addition, SSCs open the prospect of combining with other transmission technologies discussed in this section to further increase efficiency in SDM systems. 


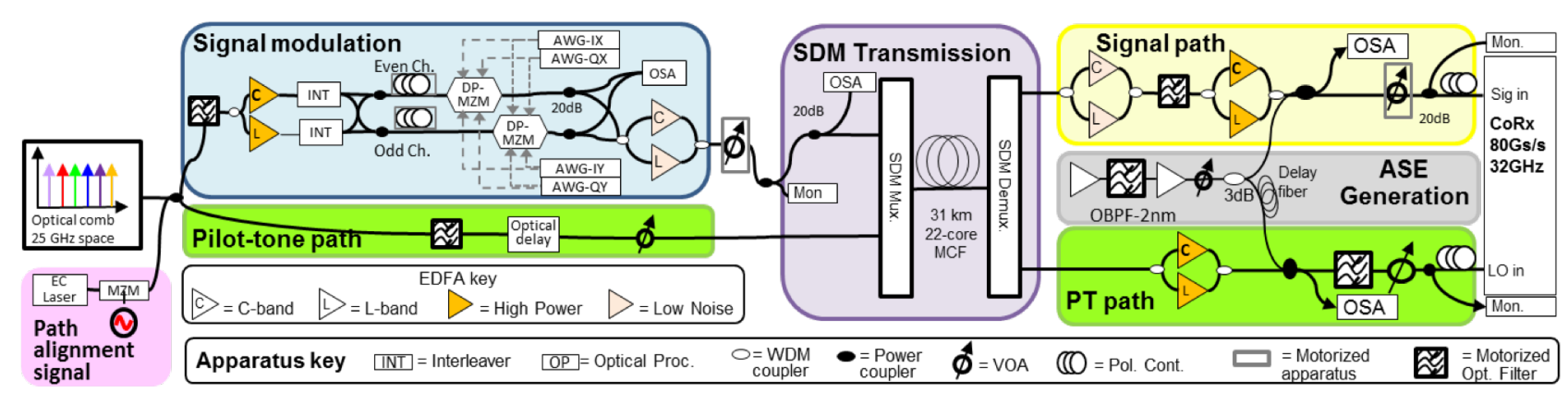

Fig. 5. Experimental set-up for intradyne and self-homodyne transmission in 22-core fiber

\section{A. Pilot-tone transmission for self-homodyne detection or shared carrier reception}

The potential for a large number of phase aligned spatial channels in SDM systems opens up the possibility of transmitting an unmodulated pilot-tone (PT) originating from the same transmission laser used to generate modulated signals on the remaining spatial channels. In shared carrier reception (SCR) the PT is received by an intradyne (ID) coherent receiver using the same local oscillator (LO) as the coherent receivers used for the signals. The precise frequency and carrier phase information extracted by the PT DSP [17] is then used to simplify the DSP for the received signal cores. Similar to the implemented polarization-multiplexed PT schemes [39], if the LO laser is sufficiently well tuned, then digital domain filtering may be used to improve the transmission characteristics. This technique was used for a long distance transmission of $10 * 128$ $\mathrm{Gb} / \mathrm{s} 16$ QAM signals with $25 \mathrm{GHz}$ spacing over a distance of $1815 \mathrm{~km}$ using $100 \mathrm{kHz}$ linewidth lasers, and allowing $1650 \mathrm{~km}$ transmission for $1 \mathrm{MHz}$ linewidth DFB laser, an increase of $165 \mathrm{~km}$ from a SCR receiver with frequency offset correction only [40].

In self-homodyne detection (SHD) systems, the PT is used as

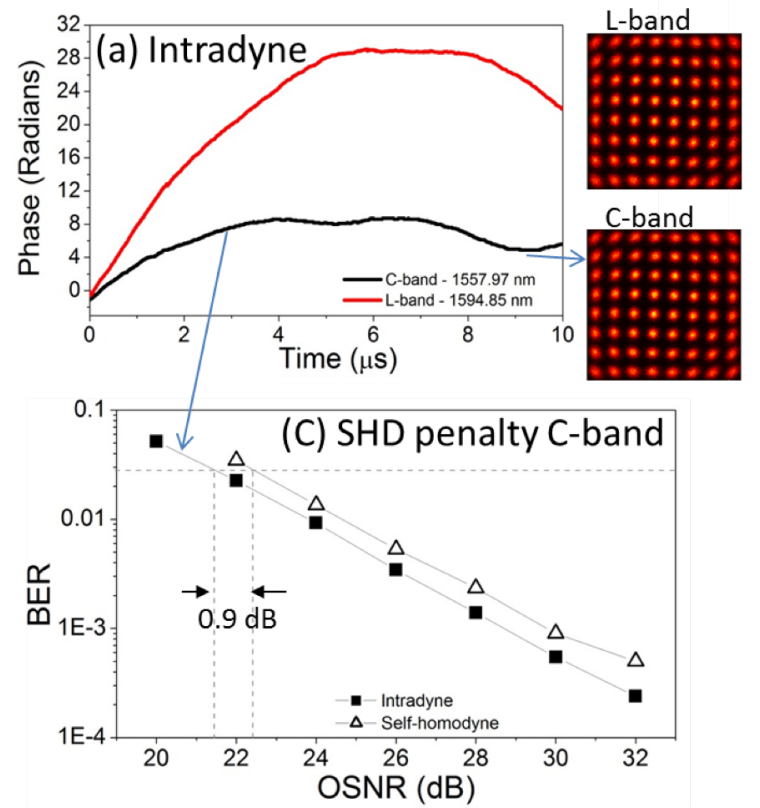

Fig. 6. Example of carrier phase evolution over 10 $\mathrm{s}$ f for (a) ID and (b) SHD and BER vs OSNR plots for ID and SHD for (c) C-band channel at $1157.97 \mathrm{~nm}$ and (d) L-band channel at $1594.85 \mathrm{~nm}$

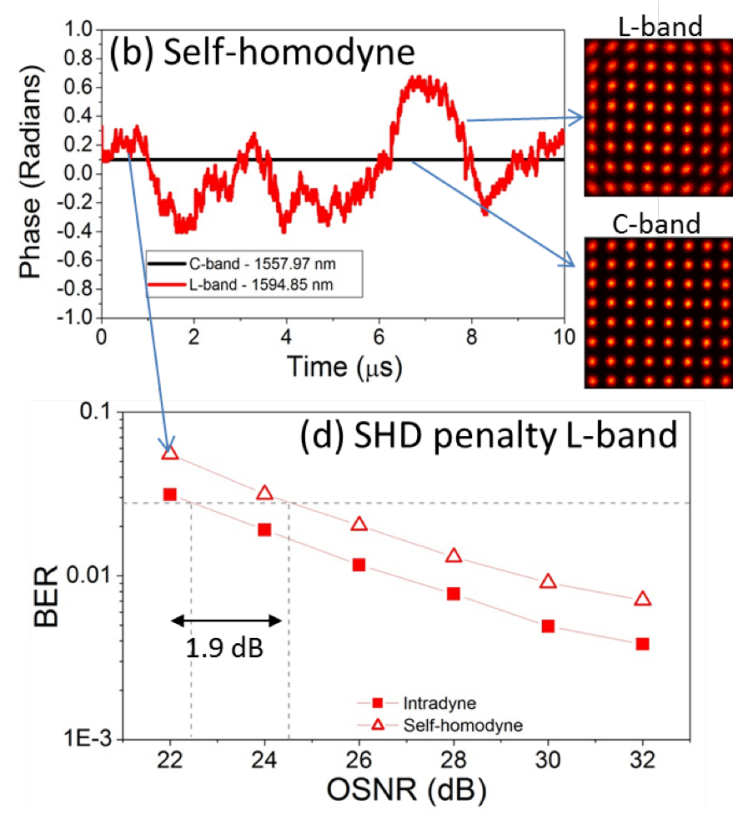

the LO laser for coherent detection of each core in order to cancel the phase noise (PN) that originates from the transmitter laser [41]. As illustrated in Fig. 4(b) for a 2 core self-homodyne $\mathrm{SSC}$, it is compatible with joint-core processing and has been shown to simplify the required DSP [42]. As with SCR, transmission of the PT reduces the SE compared to an equivalent ID system, for example, by $4.5 \%$ for a 22 -core $\mathrm{MCF}$ system. In SHD there is also an OSNR penalty arising from noise accumulation on the PT during transmission and in a WDM system the PT must be filtered to allow only a single wavelength LO. This penalty is largely determined by the ratio of PT and signal OSNR and the bandwidth of the required receiver filter in the PT path. Typical penalties for SHD demonstrations have been between 1 and $1.5 \mathrm{~dB}$ [43]. Previously, SDM-SHD has been used for high-capacity transmission with wide linewidth lasers [16], networking demonstrations [14] and in recirculating transmission where a similar distance penalty to the measured back-to back penalty was observed [44].

\section{B. Self-homodyne detection with comb transmitter}

Although the laser PN can be cancelled in an SHD system it depends on both the sensitivity of the modulation format and 
the degree of path length alignment between the signal and PT paths [43]. For the narrow linewidth comb transmitter used in [10], this was further investigated using the set-up shown in Fig. 5 and described in more detail in [45]. The comb output was first split in a $3 \mathrm{~dB}$ coupler to provide signal and PT light. On both paths, bandpass filters (BPFs) were used to select a test-channel band and WDM couplers used to direct signals to the appropriate $\mathrm{C}$ or L band EDFA. The signal path was identical to that used in [10]. After channel filtering the PT path contained additional fiber patchcords and an optical delay to allow signal-PT path length alignment using an intensity modulated signal from the free port of the PT splitting coupler. The alignment signal was able to propagate through both signal and PT paths and the resulting coherent interference was monitored at the CoRx. The delay between paths was minimized to less than 10 ps separately for C and L-band signals due to separate amplification. Individual noise loading of both signal and PT receiver inputs from a decorrelated ASE source enabled BER measurements as a function of OSNR.

Fig. 6 shows a summary of the measurements performed using both ID and SHD in the 22-core fiber set-up. Fig. 6 (a) and 6(b) show examples plots of receiver PN estimated from receiver phase compensation over $10 \mu$ s traces for $\mathrm{C}$ and L-band channels using ID and SHD respectively. In the case of ID, it is shown that the PN varies significantly along the observation interval, as a result of the phase incoherency between the transmission comb and the LO as well as some uncompensated carrier frequency offset. The average variation of 10 randomly selected measurements between C-band channels was measured to be 9.4 radians rising to 17.6 for L-band channels. In the C-band with SHD a flat phase profile was observed over the $10 \mu \mathrm{s}$. As also supported by the example constellations, this indicates good PN-cancellation (PNC) between signal and PT.

Although significantly lower than ID measurements, SHD measurements in the L-band showed some residual phase variations. The intrinsic phase noise of comb lines is determined by the noise on the comb seed laser and the distance of the broadened line form the seed wavelength resulting in higher phase for the high L-band channels over $50 \mathrm{~nm}$ from the seed. The phase variations evident in Fig. 6 appear to be at higher frequencies than in the ID case suggesting it may arise from high frequency PN components that would require a more accurate alignment of the signal and PT propagation delays to achieve PNC [30]. However, a more in depth study is required to determine this. Additional evidence of imperfect PNC in the L-band is shown in the measurement of the relative penalty of SHD compared with ID as shown in Fig. 6(c) and 6(d) for the same $\mathrm{C}$ and L-band channels respectively. The C-band measurements show a $0.9 \mathrm{~dB}$ penalty compared with ID due to degradation of transmitted PT and closely matches the theoretical prediction. However, the corresponding penalty on the L-band traces exceeds $1.9 \mathrm{~dB}$, due the uncompensated PN evident in Fig. 6(b). It should be noted that no cycle slips were observed in analyzed data and even in the L-band with ID, the primary performance limitation is ASE noise from cascaded L-band EDFAs rather than transmitter PN.

\section{MODULATION OF SPATIAL SUPER-CHANNELS}

SDM technology in conjunction with SSCs also enables greater design flexibility and greater efficiency by increasing the number of dimensions over which optical modulation is applied. Multi-dimensional modulation formats [46], [47] have previously been studied using the 4-dimensional signal space of In-Phase and Quadrature components in 2 polarizations with additional orthogonal dimensions being added by timeslots [48], wavelengths [49] or polarization states [50]. Many such formats have been proposed, typically based on geometric design methodologies such as of spherical cutting of optimal $\mathrm{N}$-dimensional lattices or by coding of a base constellation [46], [51]. Such formats offer improved SE or sensitivity, which in some cases may be flexibly implemented with the same optical hardware to respond to changing conditions. In this section, we briefly describe how some families of multi-dimensional modulation formats may be applied to SDM with a brief summary of experimental results to date and some new results.

\section{Applications to SDM}

\section{1) Space-position modulation}

In single core transmission, the most common technique for adding orthogonal dimensions is in the time domain. One common type of modulation is referred to as pulse-position modulation (PPM), which is a subset of a more general modulation scheme known as multi-dimensional position modulation (MDPM) [47]. Each dimension may be a timeslot, wavelength or SDM channel and information is coded in the presence of light in each dimension. As generalized types of MDPM, they may be simply transposed to the spatial domain as space-position modulation (SPM) using fiber modes or cores. Furthermore, each polarization may be considered individually to give space-polarization-position modulation (SPPM) such that each core has two possible slots. It has been shown that individual position modulation over polarizations has better performance in terms of both asymptotic power efficiency (APE) [51] and SE compared to position modulation over both orthogonal polarization states [47], [52].

\section{2) Set-Partitioned Modulation formats}

A number of experimental demonstrations have used advanced modulation over multiple cores in MCF. Perhaps the simplest kind of coded modulation [53] is set-partitioning (SP), where a subset of symbols is selected from a base constellation [54] by using 1 bit as a single parity-check (SPC) on all the symbol bits. For such formats, both the SE and APE, defined at high OSNR, increase with the number of cores. However, at practical OSNRs experimental implementations [18], [55] showed that increasing the number of bits over which the SPC is performed reduced the sensitivity of format. Indeed, compared to PDM-16-QAM an increase of transmission distance at a $\mathrm{BER}=1 \times 10^{-3}$ of $123 \%, 88 \%$ and $44 \%$ was measured for SPC-16QAM implemented in 1, 3 and 7 cores respectively [55].

3) Spatial-super channels with linear block codes 


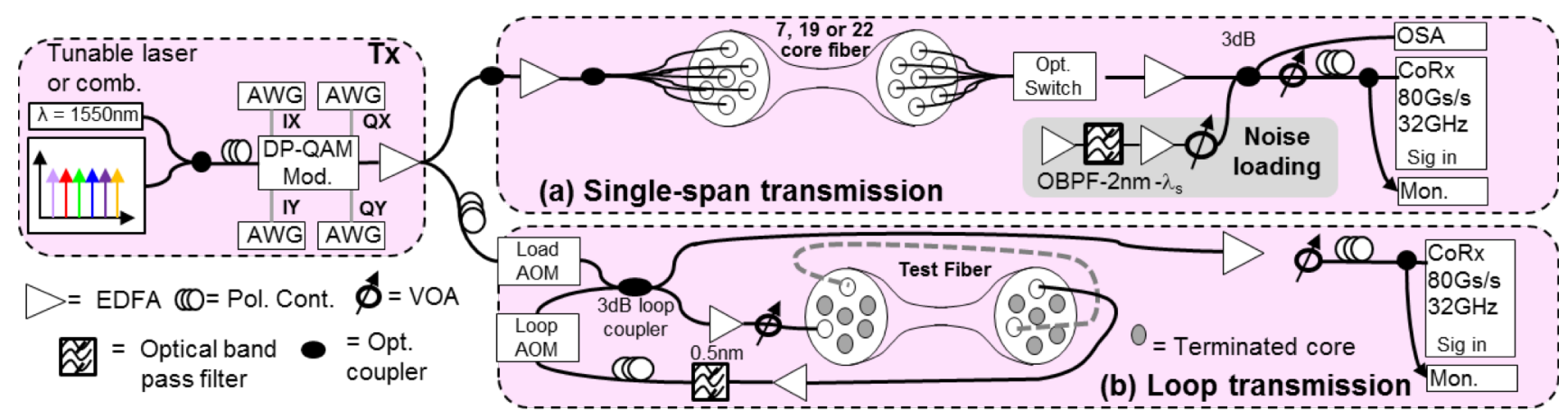

Fig. 7. Set-up for coding and modulation format experiments with (a) single-span and (b) recirculating transmission in 7, 19 and 22 core MCFs

The number of spatial channels used in research demonstrations has been steadily increasing in recent years and in 2015 exceeded 100 [7], [8]. This number of channels in combination with higher order modulation formats means that SSCs with higher bit counts per spatial symbol can be used for more complex coded modulation [53], blurring the lines between optical modulation formats and forward error correction (FEC) implemented in the optical domain. Furthermore, the drive to gain more efficiency from SDM technology and the need to compensate for imperfect properties of the optical channels has led to various approaches for incorporating elements of coding into the SDM transmission system. Multi-dimensional modulation formats based on short linear block codes (LBCs) in up to 24 dimensions was studied in [46] and experimental investigation of SSCs with LBCs of hundreds of bits [56] are described in the next section.

\section{Experimental investigation of formats for SSC}

A number of experiments of multi-core modulation formats and coding of SSCs have been performed using the set-up shown in Fig. 7. Here, we describe experimental investigations of a high sensitivity, 4-core format implemented in a 7 core fiber and coding of SSCs using 19 and 22 cores. In each case, an OSNR characterization was initially performed over a single fiber span as shown in Fig. 7(a). The transmitter output was further amplified and then split between each fiber core with different optical path lengths for signal decorrelation. In the absence of large modulator arrays, each core-specific bit sequence was received in turn with the optical switch used to select the appropriate core under test. To perform BER measurements as a function of OSNR, the signal was combined with spontaneous emission noise from a filtered EDFA and further amplified before a variable optical attenuator (VOA) for power adjustment.

Long-distance multi-core transmission measurements were emulated using a recirculating loop set-up based on a 7-core MCF as shown in Fig. 7(b). Each recirculation used 2 outer cores of a $28.35 \mathrm{~km}$ MCF span giving a total length of $56.7 \mathrm{~km}$ and round trip time of $278 \mu \mathrm{s}$. The loop contained 2 further EDFAs and a noise-limiting $100 \mathrm{GHz}$ bandwidth optical filter with optical taps and VOAs used for fiber launch power setting and monitoring. A single pair of AOM switches, with $80 \mathrm{MHz}$ frequency upshift, was used to gate loading and loop transmission, controlled by a single delay generator, which was also used for receiver triggering. The sequence corresponding to each core was loaded in turn with separate BER measurements performed as a function of transmission distance for each sequence and combined for error counting or decoding as required.

\section{1) Coded-QPSK for high sensitivity transmission}

A high-sensitivity format based on the extended Nordstrom-Robinson code [57] was experimentally investigated over 4 cores [58]. The format has 256 distinct symbols, coding 8 bits per symbol over 16 dimensions (2 quadratures in each of 2 polarizations in each of 4 cores) and therefore referred to as $8 \mathrm{~B} 16 \mathrm{D}$. While having the same spectral efficiency, its APE for the AWGN channel is $4.77 \mathrm{~dB}$ higher compared to DP-BPSK, leading to higher sensitivity. Fig. 8(a) compares the performance of the $8 \mathrm{~B} 16 \mathrm{D}$ modulation format with DP-QPSK and DP-BPSK at equal symbol rates of 25 GBaud. At a BER of $1 \times 10^{-3}$, DP-QPSK and DP-BPSK require about $13 \mathrm{~dB}$ and $10 \mathrm{~dB}$ OSNR, respectively. The $8 \mathrm{~B} 16 \mathrm{D}$ modulation format, however, only requires about 7.5 dB OSNR at equal bit-rate as DP-BPSK. The transmission reach was also
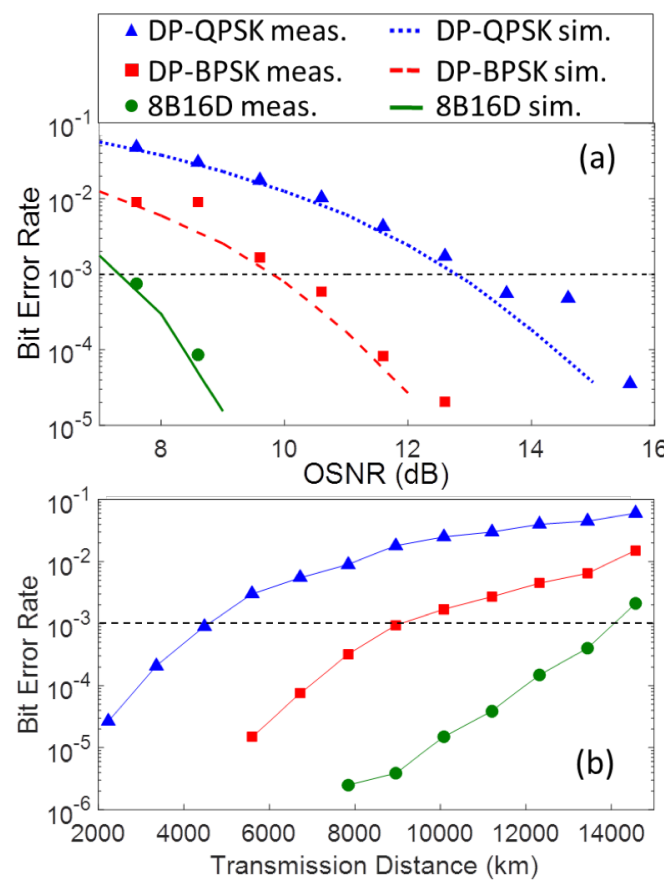

Fig. 8. (a) BER vs OSNR after single span and (b) BER vs transmission distance for PDM-BPSK, PDM-QPSK and 8B16D format [60] 

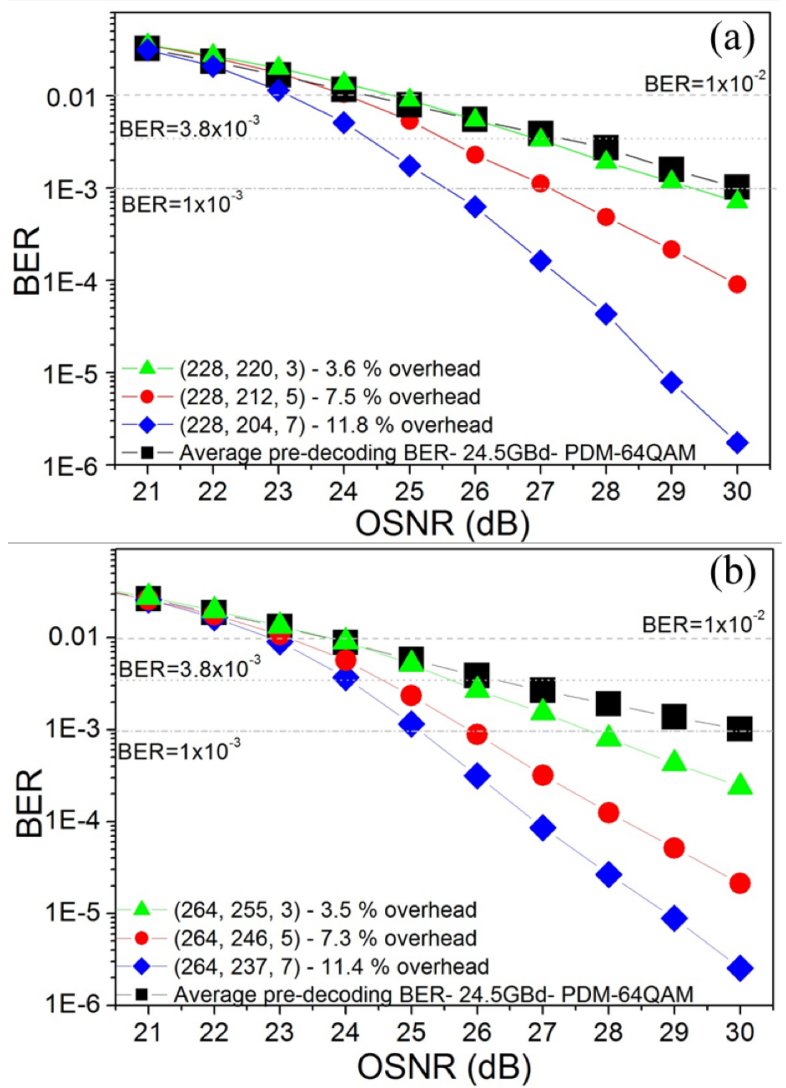

Fig. 9. BER vs OSNR after single span transmission of (a) 19-core [57] and (b) 22-core [18] LBC-SSC

investigated with the same formats as shown in Fig. 8(b) showing an increased reach of $55 \%$ and $300 \%$ over DP-BPSK and QPSK respectively at $\mathrm{BER}=1 \times 10^{-3}$. Being a coded sub-set of QPSK, this modulation format requires the same optical transmitter and receiver hardware. Thus, it is potentially useful as a simple method of extending the reach of QPSK based transmission where $50 \%$ of the SE can be traded for $300 \%$ increased distance at $\mathrm{BER}=1 \times 10^{-3}$

\section{2) Implementing spatial LBCs}

An $(n, k, d)$ LBC takes $k$-bit messages and converts them into $n$-bit codewords with a code rate $k / n$ and overhead $n / k-1$. This is done by appending $n-k$ parity bits to each block of $k$ message bits to maximize the minimum Hamming distance $d$. A hard-decision decoder can correct up to $t=(d-1) / 2$ if $d$ is odd and $(d-2) / 2$ errors if $d$ is even. The modulation of PDM-64QAM allows transmission of 12 bits/core, giving $n=$ 228 bits for 19-core SSCs and $n=264$ bits for 22-core SSCs. Since the classical code families do not provide codes with those lengths $n$, we used a technique referred to as code shortening [59], which means that information bits are removed from a longer code. If an $(n, k, d) \mathrm{LBC}$ is shortened with $s$ bits, the resulting code has parameters $(n-s, k-s, d)$. Specifically, for the 19-core case we designed codes with parameters $(228,220,3),(228,212,5)$, and $(228,204,7)$ by shortening BoseChaudhury-Hocquenghem $(\mathrm{BCH})$ codes [59] with parameters $(255,247,3),(255,239,5)$, and $(255,231,7)$, respectively. The shortened codes have overheads $3.6 \%, 7.5 \%$, and $11.8 \%$, respectively, and are the best known codes with these parameters (highest $k$ for given $n$ and $d$ ) [60]. For 22-core SSCs, codes with $(n, k, d)=(264,255,3),(264,246,5)$, and $(264,237,7)$ were similarly constructed from length-511 BCH codes, having overheads of $3.5 \%, 7.3 \%$ and $11.4 \%$, respectively. For shorthand we refer to each code by their Hamming distance as $\mathrm{H} 3, \mathrm{H} 5$ and $\mathrm{H} 7$ codes. For experiments, each coded data block $\mathbf{x}$ was generated according to $\mathbf{x}=\mathbf{u} \cdot \mathbf{G}$, where $\mathbf{u}$ is a $k$-bit block of randomly generated data, $\mathbf{G}$ is the code's generator matrix, and the matrix multiplication is carried out modulo 2. $\mathbf{x}$ was then divided into 6-bit sections, with each section mapped onto a 64QAM symbol using Gray mapping, for modulation onto each polarization component of each fiber core. This process was repeated until reaching $2^{15}-1$ multi-core symbols for experimental investigation. At the receiver, the coded data blocks were reassembled from the received data streams of each fiber core after hard detection and then decoded by syndrome decoding [59]. This is a very fast decoding technique, at the cost of a high memory overhead. The syndromes are located from a pre-computed syndrome table, whose size scales as $2^{n-k}$, using a bisection search. The BER of the whole decoded data sequence could then be compared.

\section{3) Results of $L B C$ experiments}

Fig. 9 (a) and (b) show the OSNR characterization after single span transmission for the 19-core and 22-core LBC-SSCs respectively and show each of the 3 codes. Also shown is the average BER of the transmitted sequence before decoding. Both plots show similar trends with OSNR improvement compared to uncoded PDM-64QAM increasing for lower BER thresholds with any benefit likely to be at BERs typical of HD-FEC systems or lower. At $\mathrm{BER}=1 \times 10^{-3}$, a reduction in required OSNR of $1.2 \mathrm{~dB}, 2.4 \mathrm{~dB}$, and $3.2 \mathrm{~dB}$ is seen for the $\mathrm{H} 3, \mathrm{H} 5$, and $\mathrm{H} 7$ codes, respectively for 19-SSCs and $2.2 \mathrm{~dB}, 4.1 \mathrm{~dB}$, and $5 \mathrm{~dB}$ for the 22-core case. At $\mathrm{BER}=1 \times 10^{-2}$, close to input BER of typical soft-decision FEC systems, the required OSNR of the $\mathrm{H} 3, \mathrm{H} 5$ and $\mathrm{H} 7$ codes becomes $-0.3 \mathrm{~dB}$, $0.3 \mathrm{~dB}$, and $1.1 \mathrm{~dB}$ for 19 cores and $0 \mathrm{~dB}, 0.6 \mathrm{~dB}$, and $0.9 \mathrm{~dB}$ for 19 and 22 cores respectively. Whilst these results show that these particular codes are not very competitive compared with systems with an outer soft-decision FEC code, they do serve to illustrate the principle that SSC coding can be used to trade some SE for required OSNR with the potential to equalize

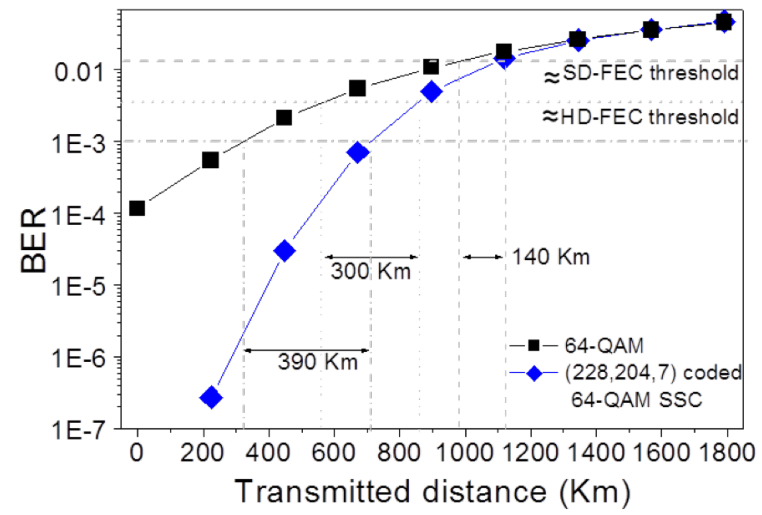

Fig. 10. Transmitted distance for PDM-64QAM before decoding and 228. 204. 7 PDM-64OM LBC-SSC $\lceil 57\rceil$ 


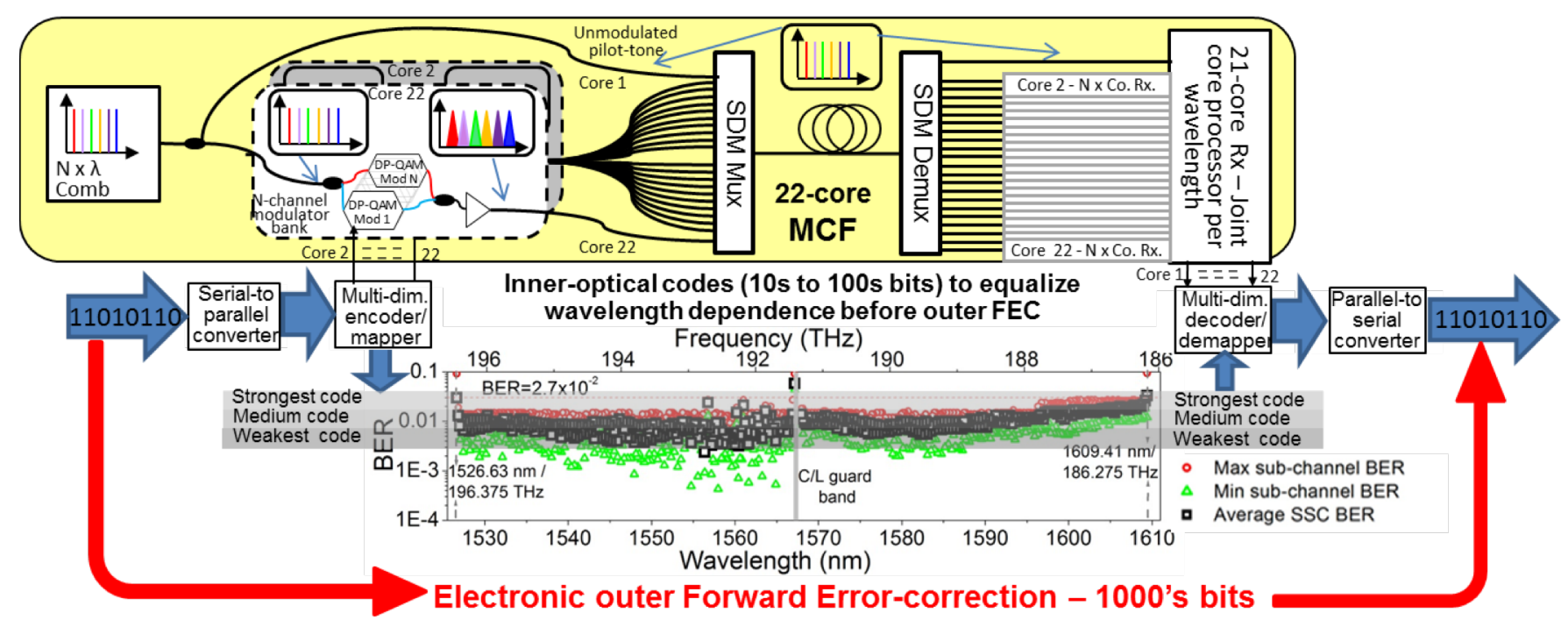

Fig. 11. Schematic of high-capacity MCF link using 22-core fiber and BER summary (lower Inset) showing the maximum, minimum and average sub-channel BER for each 22-core spatial-super channel

channels of varying performance.

Fig. 10 summarizes the recirculating transmission experiment comparing the $\mathrm{H} 7$ code over 19-core SSCs and the average pre-decoding BER for the optimal fiber launch power of $0 \mathrm{dBm}$. Again, at high BERs, the coded and pre-decoding BERs converge with only a $18 \%$ reach improvement at $\mathrm{BER}=1 \times 10^{-2}$ but increasing to $54 \%$ and $122 \%$ for $\mathrm{BER}=3.8 \times 10^{-3}$ and $\mathrm{BER}=1 \times 10^{-3}$ respectively. Assuming a pre-FEC BER threshold of $3.8 \times 10^{-3}$, this result shows that such a code can extend the reach of a system with HD-FEC from $560 \mathrm{~km}$ to $870 \mathrm{~km}$. In addition, such codes might be used for equalizing the transmission distance of different WDM channels. This result further suggests that by carefully selecting appropriate codes for an inner optical FEC code and an outer HD-FEC, it may be possible to achieve comparable error correcting power of SD-FEC. For example, Fig. 9(a) and Fig. 10 show that in the receiver OSNR range of $24.3-27.3 \mathrm{~dB}$ or the transmission range of $560-870 \mathrm{~km}$, the $\mathrm{H} 7$ code reduces the BER to below the usual threshold for HD-FEC $\left(3.8 \times 10^{-3}\right)$, meaning that a post-FEC BER in the region of $1 \times 10^{-15}$ may be achieved with an additional $7 \%$ overhead on the $11.8 \%$ code overhead to give a total FEC overhead of $19.3 \%$. Such overhead is similar to a typical SD-FEC overhead of $20 \%$ but maybe achieved without incurring the additional hardware costs and complexity required for SD-FEC implementation.

\section{COdED SSCS IN A High CAPACITY MCF SyStem}

Finally, in this section we present a speculative MCF system with reference to a transmission experiment based on a homogeneous SM-MCF and discuss how such a system may be combined with technologies discussed in the previous section. Transmission capacity exceeding $2 \mathrm{~Pb} / \mathrm{s}$ was demonstrated by exploiting $<80 \mathrm{~nm}$ transmission bandwidth, enabled by a wideband optical comb source in combination with high spectral efficiency modulation of 22-core SSCs [10]. Fig. 11 shows a schematic of how such a transmitter and fiber could be integrated with technologies such as joint-core reception, SHD and SSC coding. The inset shows the measured maximum, minimum and average BER of each of the 22-core SSCs from [10]. From the BER plot, it is evident that the uncoded channel has strong wavelength dependence with individual sub-channel BERs varying by over 3 orders of magnitude. Such variation can be problematic for choosing the most efficient outer FEC per channel since the overhead is usually determined by the pre-FEC and required post-FEC BER [61]. The possibility illustrated in Fig. 11 is to use some coding overhead on groups of channels, such as across SSCs, to reduce the variation of pre-FEC BERs as shown by the lower variance in average SSC BER (black squares). Furthermore, depending on the quality of each SSC, specific codes may be used to reach the target pre-FEC BER with the smallest possible overhead. Although not necessarily the optimum grouping strategy, in this example SSCs allow short optical codes of 100 s of bits to condition the pre-FEC BERs of serially coded electronic forwarderror-correction using 1000 s bits to try to maximize the overall throughput.

\section{CONCLUSION}

We have described a range of system technologies compatible with spatial super-channel transmission in SDM systems. While potentially applicable to other forms of SDM, they are particularly suited to homogeneous, single-mode (SM)-multi-core fibers (MCF) transmission, exploiting the low variation in propagation delay between spatial channels. Few-mode MCFs may be able offer increased capacity over short distances, however, SM-MCFs are likely to be more suitable for longer distances and since they require less drastic changes to transmitter and receiver hardware, offering a simpler migration path from currently installed systems. The extent to which inter-core crosstalk may impair long-haul transmission is not yet clear. Although fibers with crosstalk lower than the level measured to limit transmission distance have been reported, the impact of dynamic time and frequency fluctuations, bending radius and temperature require further study. 


\section{ACKNOWLEDGMENTS}

The wide-band comb and the 22-core MCF were produced by RAM Photonics and Sumitomo Electric Industries respectively. We also thank the NICT technical staff.

\section{REFERENCES}

[1] E. B. Desurvire, "Capacity Demand and Technology Challenges for Lightwave Systems in the Next Two Decades," J. Lightw. Technol., vol. 24, no. 12, pp. 4697-4710, Dec. 2006.

[2] D. J. Richardson, J. M. Fini, and L. E. Nelson, "Space-division multiplexing in optical fibres," Nat. Photonics, vol. 7, no. 5, pp. 354-362, Apr. 2013.

[3] G. M. Saridis, D. Alexandropoulos, G. Zervas, and D. Simeonidou, "Survey and Evaluation of Space Division Multiplexing: From Technologies to Optical Networks," IEEE Commun. Surv. Tutor., vol. 17, no. 4, pp. 2136-2156, 2015.

[4] K. Imamura, K. Mukasa, and T. Yagi, "Effective space division multiplexing by multi-core fibers," in Proc. Eur. Conf. Opt. Сотти., Torino, Italy, Sept, 2010, p. Th4C.3.

[5] R. Ryf, "SDM components and MIMO experiments in multimode and multicore fibers," in Proc. Eur. Conf. Opt. Commu., Cannes, France, Sept, 2014, p Th.2.1.1.

[6] N. Bozinovic, Y. Yue, Y. Ren, M. Tur, P. Kristensen, AE Willner, S. Ramachandran, "Orbital angular momentum (OAM) based mode division multiplexing (MDM) over a Km-length fiber," in Proc. Eur. Conf. Opt. Commu., Amsterdam, Netherlands, Sept, 2012, p Th.3.C.6.

[7] J. Sakaguchi, W. Klaus, J.-M. Delgado Mendinueta, B.J. Puttnam, R.S Luis, Y. Awaji, N. Wada, T. Hayashi, T. Nakanishi, T. Watanabe, Y Kokubun, T. Takahata, and T. Kobayashi, "Large spatial channel (36-Core $\times 3$ mode) heterogeneous few-mode multicore fiber, " J. Lightw. Technol., vol. 34, no.1, pp. 93-103, 2016.

[8] K. Igarashi, D. Souma, Y. Wakayama, K. Takeshima, Y. Kawaguchi, T. Tsuritani, I. Morita, and M. Suzuki, "114 pace-Division-Multiplexed Transmission over $9.8-\mathrm{km}$ Weakly-Coupled-6-Mode uncoupled -19Core Fibers," in Optical Fiber Communication Conference, 2015, p. Th5C-4.

[9] D. Soma, K. Igarashi, Y. Wakayama, K. Takeshima, Y. Kawaguchi, N. Yoshikane, T. Tsuritani, I. Morita, M. Suzuki, "2.05 Peta-bit/s super-nyquist-WDM SDM transmission using 9.8-km 6-mode 19-core fiber in full C band," in Proc. Eur. Conf. Opt. Commu., Valencia, Spain, Sept, 2012, PDP3-1.

[10] B. J. Puttnam, R.S. Luís, W. Klaus, J. Sakaguchi, J.-M. Delgado Mendinueta, Y. Awaji, N. Wada, Y. Tamura, T. Hayashi, M. Hirano, J. Marciante., " $2.15 \mathrm{~Pb} / \mathrm{s}$ transmission using a 22 core homogeneous single-mode multi-core fiber and wideband optical comb," in Proc. Eur. Conf. Opt. Coтmu., Valencia, Spain, Sept, 2012, PDP3-1

[11] I. Morita, K. Igarashi, H. Takahashi, T. Tsuritani, and M. Suzuki, "Trans-oceanic class ultra-long-haul transmission using multi-core fiber," Opt. Express, vol. 22, no. 26, p. 31761, Dec. 2014.

[12] Y. Lee, K. Tanaka, E. Nomoto, H. Arimoto, and T. Sugawara, "Multi-core fiber technology for optical-access and short-range links," in 2014 12th International Conference on Optical Internet 2014 (COIN), 2014, pp. 1-2.

[13] K. Tanaka, Y. Lee, E. Nomoto, T. Sugawara, and H. Arimoto, "Automatic Impairment-Aware Optical Path Switching in Multicore Fiber Link Based on Multiring Structure," J. Light. Technol., vol. 33, no. 14, pp. 3060-3068, Jul. 2015.

[14] N. Amaya, S. Yan, M. Channegowda, B.R. Rofoee, Y. Shu, M. Rashidi, Y. Ou, E. Hugues-Salas, G. Zervas, R. Nejabati, D. Simeonidou, B.J. Puttnam, W. Klaus, J. Sakaguchi, T. Miyazawa, Y. Awaji, H. Harai, and $\mathrm{N}$. Wada, "Software defined networking (SDN) over space division multiplexing (SDM) optical networks: features, benefits and experimental demonstration," Opt. Express, vol. 22, no. 3, pp. 36383647, Feb, 2014.

[15] M. Feuer, L. Nelson, X. Zhou, S.Woodward, R. Isaac, B. Zhu, T. Taunay, M. Fishteyn, J. Fini, and M. Yan, "Joint digital signal processing receivers for spatial superchannels," IEEE Photon. Technol. Lett., vol. 24, no. 21, pp. 1957-1960, Nov. 2012

[16] B. J. Puttnam,R.Luis, J.-M.Delgado-Mendinueta, J. Sakaguchi, W.Klaus, Y. Awaji, N. Wada, A. Kanno, and T. Kawanishi, "High-capacity self-homodyne PDM-WDM-SDM transmission in a 19-core fiber," Opt. Exp.,vol. 22, no. 18, pp. 21185-21191, Sep. 2014

[17] E. L. T. de Gabory, M. Arikawa, Y. Hashimoto, T. Ito, and K. Fukuchi, "A shared carrier reception and processing scheme for compensating frequency offset and phase noise of space-division multiplexed signals over multicore fibers," in Optical Fiber Communication Conference, 2013, p. OM2C-2

[18] B. J. Puttnam, T. A. Eriksson, J.-M. D. Mendinueta, R. S. Luis, Y. Awaji, N.Wada,M. Karlsson, and E. Agrell, "Modulation formats for multi-core fiber transmission," Opt. Exp., vol. 22, no. 26, pp. 32457-32469, Dec. 2014.
[19] B. J. Puttnam, R. S. Luís, J. Sakaguchi, W Klaus, J-M, Delgado Mendinueta, Y. Awaji, N. Wada, and E. Agrell., "Pb/s, Homogeneous, Single-mode, Multi-Core Fiber Systems," Eur. Conf. Opt. Comms, Dusseldorf, Germany,Tu.1.D.2, 2016.

[20] T. Hayashi, T. Taru, O. Shimakawa, T. Sasaki, and E. Sasaoka, "Design and fabrication of ultra-low crosstalk and low-loss multi-core fiber," Opt. Express, vol. 19, no. 17, pp. 16576-16592, 2011.

[21] R. S. Luís, B. J. Puttnam, A. Cartaxo, W. Klaus, J-M. Delgado Mendinueta Y. Awaji, N. Wada, T. Nakanishi, T. Hayashi and T. Sasaki, "Time and Modulation Frequency Dependence of Crosstalk in Homogeneous Multi-Core Fibers," J. Light. Technol., vol. 34, no. 2, pp. 441-447, Jan. 2016.

[22] J. M. Fini, B. Zhu, T. F. Taunay, M. F. Yan, and K. S. Abedin, "Statistical Models of Multicore Fiber Crosstalk Including Time Delays," J. Light. Technol., vol. 30, no. 12, pp. 2003-2010, Jun. 2012.

[23] A. V. T. Cartaxo, R. S. Luís, B. J. Puttnam, T. Hayashi, Y. Awaji, and N. Wada, "Dispersion Impact on the Crosstalk Amplitude Response of Homogeneous Multi-Core Fibers," IEEE Photonics Technol. Lett., vol. 28 , no. 17 , pp. 1858-1861, Sep. 2016.

[24] P. Winzer, A. Gnauck, A. Konczykowska, F. Jorge, and J.-Y. Dupuy, "Penalties from in-band crosstalk for advanced optical modulation formats," in Proc. Eur. Conf. Opt. Commu., Cannes, France, Sept, 2014, p. $\mathrm{Tu}-5$.

[25] B. J. Puttnam, R. S. Luís, T.E Eriksson, W. Klaus, J.-M. Delgado Mendinueta, Y. Awaji, N. Wada, "Impact of Inter-Core Crosstalk on the Transmission Distance of QAM Formats in Multi-Core Fibers," IEEE Photonics J., vol. 8, no. 2, 2016.

[26] K. Takenaga Y. Arakawa, S. Tanigawa, N. Guan, S. Matsuo, K.Saitoh, and M. Koshiba, "Reduction of crosstalk by trench-assisted multi-core fiber," in Optical Fiber Communication Conference, 2011, p. OWJ4.

[27] Y. Sasaki, K. Takenaga, S. Matsuo, K. Saitoh, and M. Koshiba, "Trench-assisted low-crosstalk few-mode multicore fiber," in Proc. Eur. Conf. Opt. Commu., London, UK, Sept, 2013, p. Mo.3.A.5.

[28] A. Sano, H. Takara, T. Kobayashi, and Y.Miyamoto, "Crosstalkmanaged high capacity long haul multicore fiber transmission with propagation direction interleaving," J. Lightw. Technol., vol. 32, no. 16, pp. 2771-2779, Aug. 2014.

[29] M. Koshiba, K. Saitoh, and Y. Kokubun, "Heterogeneous multi-core fibers: proposal and design principle," IEICE Electron. Express, vol. 6, no. 2, pp. 98-103, 2009.

[30] T. Hayashi, T. Nakanishi, F. Sato, T. Taru, and T. Sasaki, "Characterization of interconnect multi-core fiber cable: Mechanical/thermal characteristics and inter-core crosstalk of the straightened cable," in Proc, optical interconnects 2016, pp. 92-93.

[31] R. S. Luis, B. J. Puttnam, J. M. Delgado Mendinueta, Y. Awaji, and N. Wada, "Impact of spatial channel skew on the performance of spatial-division multiplexed self-homodyne transmission systems," in Photonics in Switching (PS), 2015 International Conference on, 2015, pp. 37-39.

[32] G. M. Saridis, B. J. Puttnam, R. S. Luís, W. Klaus, Y. Awaji, G. Zervas, D. Simeonidou and N. Wada., "Dynamic Skew Measurements in 7, 19 and 22-core Multi Core Fibers," in optoelectronics and communicatiosn conference 2016 paper MC2-1

[33] R. S. Luís, B. J. Puttnam, J. M. D. Mendinueta, W. Klaus, Y. Awaji, and N. Wada, "Comparing inter-core skew fluctuations in multi-core and single-core fibers," in 2015 Conference on Lasers and Electro-Optics (CLEO), 2015, p. SM2L.5.

[34] J. Sakaguchi, W. Klaus, B. J. Puttnam, J-M Delgado Mendinueta, Y. Awaji, Naoya Wada, Y. Tsuchida, K. Maeda, M. Tadakuma, K. Imamura, R. Sugizaki, T. Kobayashi, Y. Tottori, M. Watanabe, and R. V. Jensen, "19-core MCF transmission system using EDFA with shared core pumping coupled via free-space optics," Opt. Express, vol. 22, no. 1, p. 90, Jan. 2014

[35] B. P.-P. Kuo, E. Myslivets, V. Ataie, E. G. Temprana, N. Alic, and S. Radic, "Wideband parametric frequency comb as coherent optical carrier," J. Light. Technol., vol. 31, no. 21, pp. 3414-3419, 2013.

[36] V. Ataie, E Temprana, L Liu, E Myslivets, BPP Kuo, N Alic, S Radic, "Ultrahigh Count Coherent WDM Channels Transmission Using Optical Parametric Comb-Based Frequency Synthesizer," J. Light. Technol., vol. 33, no. 3, pp. 694-699, Feb. 2015.

[37] L.E. Nelson, M.D. Feuer, K. Abedin, X. Zhou, T.F. Taunay, J.M. Fini, B. Zhu, R. Isaac, R. Harel, G. Cohen, and D.M. Marom,, "Spatial Superchannel Routing in a Two-Span ROADM System for Space Division Multiplexing," J. Light. Technol., vol. 32, no. 4, pp. 783-789, Feb. 2014.

[38] M.D. Feuer, L.E. Nelson, K. Abedin, X. Zhou, T.F. Taunay, J.F. Fini, B. Zhu, R. Isaac, R. Harel, G. Cohen, and D.M. Marom, "ROADM system for space division multiplexing with spatial superchannels," in Optical Fiber Communication Conference, 2013, p. PDP5B-8.

[39] R. S. Luís, B.J Puttnam, J.-M. Mendinueta, S. Shinada, M. Nakamura, Y. Kamio, and N. Wada, "Digital Self-Homodyne Detection," IEEE Photonics Technol. Lett., vol. 27, no. 6, pp. 608-611, Mar. 2015.

[40] E. L. T. de Gabory, M. Arikawa, T. Ito, and K. Fukuchi, "DWDM transmission of $128 \mathrm{~Gb} / \mathrm{s}$ PM-16QAM signal over $1815 \mathrm{~km}$ of 7 -core MCF using shared carrier reception for improving the received signal quality," in Proc. Eur. Conf. Opt. Commu., London, UK, Sept, 2013, p We.2.D.4 
[41] P. Johannisson, M. Sjodin, M. Karlsson, E. Tipsuwannakul, and P. Andrekson, "Cancellation of Nonlinear Phase Distortion in Self-Homodyne Coherent Systems," IEEE Photonics Technol. Lett., vol. 22 , no. 11 , pp. $802-804$, Jun. 2010.

[42] J-M. Delgado Mendinueta, B.J. Puttnam, J.Sakaguchi, W.Klaus, Y.Awaji, N.Wada, A.Kanno and T.Kawanishi, "Investigation of Receiver DSP Carrier Phase Estimation Rate for Self-homodyne Space-division Multiplexing Communication Systems," in Optical Fiber Communication Conference, 2013, p. JTh2A.

[43] R.S.Luis, B.J.Puttnam, J-M. Delgado Mendinueta, W.Klaus, J. Sakaguchi, Y.Awaji, A.Kanno, T.Kawanishi and N.Wada, "OSNR Penalty of Self-Homodyne Coherent Detection in Spatial-DivisionMultiplexing Systems," IEEE Photonics Technol. Lett., vol. 26, no. 5, pp. 477-479, Mar. 2014

[44] B.J. Puttnam, R. Luis, J.-M. Delgado Mendinueta, J. Sakaguchi, W. Klaus, Y. Awaji, N. Wada, A. Kanno, and T. Kawanishi, "Long distance transmission in a multi-core fiber with self-homodyne detection," in Optical Fiber Communications Conference and Exhibition (OFC), 2015, 2015, p. Th1D.5.

[45] B. J. Puttnam, R. S. Luís, Y. Awaji and N. Wada. "Self-Homodyne and Phase Measurements for MCF Transmission with Wideband Comb Transmitter" IEEE Photonics Conference Paper TuB2.2 (2016)

[46] D. S. Millar, T. Koike-Akino, S.Ö. Arrk, K. Kojima K. Parsons T. Yoshida T. Sugihara "High-dimensional modulation for coherent optical communications systems," Opt. Express, vol. 22, no. 7, p. 8798, Apr. 2014.

[47] T. A. Eriksson, P. Johannisson, B. J. Puttnam, E. Agrell, P. A. Andrekson, and M. Karlsson, "K-Over-L Multidimensional Position Modulation," J. Light. Technol., vol. 32, no. 12, pp. 2254-2262, 2014.

[48] H. Sugiyama and K. Nosu, "MPPM: a method for improving the band-utilization efficiency in optical PPM," J. Light. Technol., vol. 7, no. 3, pp. 465-472, Mar. 1989.

[49] T. A. Eriksson, P. Johannisson, M. Sjödin, E. Agrell, P. A. Andrekson, and M. Karlsson, "Frequency and polarization switched QPSK," in Proc. Eur. Conf. Opt. Commu., London, UK, Sept, 2013, p. Th.2.D.4.

[50] H. Bulow, "Polarization QAM modulation (POL-QAM) for coherent detection schemes," in Conference on Optical Fiber Communications 2009, p. OWG2.

[51] E. Agrell and M. Karlsson, "Power-Efficient Modulation Formats in Coherent Transmission Systems," J. Light. Technol., vol. 27, no. 22, pp. 5115-5126, Nov. 2009.

[52] M. Karlsson and E. Agrell, "Generalized pulse-position modulation for optical power-efficient communication," Proc. Eur. Conf. Opt. Commu., Cannes, France, Sept, 2014, p. Tu.6.B.6.

[53] G. Ungerboeck, "Channel coding with multilevel/phase signals," IEEE Trans. Inf. Theory, vol. 28, no. 1, pp. 55-67, Jan. 1982.

[54] T. A. Eriksson, R. S. Luís, B. J. Puttnam, J-M. Delgado Mendinueta, M. Karlsson, P. A. Andrekson, Y. Awaji, N. Wada and E. Agrell, "Single parity check-coded 16QAM over spatial superchannels in multicore fiber transmission," Opt. Express, vol. 23, no. 11, pp. 14569-14582, Jun. 2015.

[55] L. Beygi, E. Agrell, J. M. Kahn, and M. Karlsson, "Coded Modulation for Fiber-Optic Networks: Toward better tradeoff between signal processing complexity and optical transparent reach," IEEE Signal Process. Mag., vol. 31, no. 2, pp. 93-103, Mar. 2014.

[56] B. J. Puttnam, R. S. Luis, J. M. D. Mendinueta, Y. Awaji, N. Wada, and E. Agrell, "Linear block-coding across $>5 \mathrm{~Tb} / \mathrm{s}$ PDM-64QAM spatialsuper-channels in a 19-core fiber," in Proc. Eur. Conf. Opt. Commu., Valencia, Spain, Sept, 2012, p P.5.6.

[57] A. W. Nordstrom and J. P. Robinson, "An optimum nonlinear code," Inf. Control, vol. 11, no. 5, pp. 613-616, Nov. 1967.

[58] G. Rademacher, B. J. Puttnam, R. S. Luis, Y. Awaji, N. Wada, E. Agrell and K. Petermann, "Experimental investigation of a 16-dimensional modulation format for long-haul multi-core fiber transmission," in Proc. Eur. Conf. Opt. Commu., Valencia, Spain, Sept, 2012, p P.5.10

[59] G. Clark. Jr and J. B. Cain, "Group Codes," in Error-Correction Coding for Digital Communications, Springer US, 1981, pp. 49-96.

[60] M. Grassl, "Code tables: Bounds on the parameters of various types of codes", online, codetables.de.

[61] L. M. Zhang and F. R. Kschischang, "Staircase Codes With 6\% to 33\% Overhead," J. Lightw. Technol., vol. 32, no. 10, pp. 1999-2002, May 2014.

Ben Puttnam (M'12) received the MPhys degree in Physics from the University of Manchester (UK) in 2000 and the $\mathrm{PhD}$ degree from University College London in 2008. In between he was working for over 2 years as a Network Engineer for T-mobile (UK). From October 2008, he spent 9 months in the Photonic Network System Laboratory at the National Institute of Information and Communications Technology (NICT) in Tokyo, Japan, supported by JSPS. After a 5 month stay in the Photonics group at Chalmers University, Göteborg, Sweden supported by the Ericsson research foundation he re-joined NICT in March 2010. His current research interests are applications and sub-systems for SDM transmission and phase sensitive amplifier applications using PPLN waveguides.

Ruben S. Luís received is degree and M. Sc. in electrical and computer engineering from Instituto Superior Tecnico, Technical University of Lisbon in 2000 and 2003, respectively, and is Ph.D. In electrical engineering from the University of Aveiro in 2007. Since 2016, he has been with the Photonic Network System Laboratory of the National Institute of Information and Communications Technology, Tokyo, Japan, where he researches advanced coherent transmission systems using spatial division multiplexing and optical packet switching.

Georg Rademacher received the Dipl.-Ing. and Dr.-Ing. degree in electrical engineering from Technische Universitaet Berlin, Germany, in 2011 and 2015 respectively. In 2016, he joined the photonic network system laboratory at the National Institute of Information and Communications Technology (NICT) in Tokyo, Japan, where he is engaged in research on space division multiplexed transmission systems.

Jun Sakaguchi (M'10) received the B.S., M.S., and Ph.D. degrees in the field of high-energy physics from the University of Tokyo, Tokyo, Japan, in 1998, 2000, and 2003, respectively. $\mathrm{He}$ received the next Ph.D. degree in electronics engineering from University of Electro-Communications, Tokyo, Japan, in 2008 after working on SOA-based wavelength converters.

He was a post-doctoral fellow at Nara Institute of Science and Technology (NAIST), Ikoma, Japan from 2008 to 2010, where he worked for modeling, design, characterization and demonstration of VCSEL-based high-speed optical buffer memories. He was a post-doctoral fellow at the National Institute of Information and Communications Technology (NICT) from 2010 to 2014, where he worked for large-scale space division multiplexed fiber-optic communication systems, high-coherency lightwave distribution, a special kind of real-time digital signal processing and applications of optical frequency comb. Since 2014 he has been a permanent staff at the NICT. He has published 80 papers in refereed English journals and international conferences.

Dr. Sakaguchi is a member of the IEEE Photonics Society.

Erik Agrell received the Ph.D. degree in information theory in 1997 from Chalmers University of Technology, Sweden. From 1997 to 1999, he was a Postdoctoral Researcher with the University of California, San Diego and the University of Illinois at Urbana-Champaign. In 1999, he joined the faculty of Chalmers University of Technology, where he is a Professor in Communication Systems since 2009. In 2010, he cofounded the Fiber-Optic Communications Research Center (FORCE) at Chalmers, where he leads the signals and systems research area. $\mathrm{He}$ is a Visiting Professor at University College London since 2014. His research interests belong to the fields of information theory, coding theory, and digital communications, and his favorite applications are found in optical communications.

Prof. Agrell served as Publications Editor for the IEEE Transactions on Information Theory from 1999 to 2002 and as 
Associate Editor for the IEEE Transactions on Communications from 2012 to 2015 . He is a recipient of the 1990 John Ericsson Medal, the 2009 ITW Best Poster Award, the 2011 GlobeCom Best Paper Award, the 2013 CTW Best Poster Award, and the 2013 Chalmers Supervisor of the Year Award.

Werner Klaus received the M.Sc. degree in electrical engineering/communications engineering from the Vienna University of Technology, Vienna, Austria in 1991, and the Ph.D. degree in electronics engineering from the University of Tokyo, Tokyo, Japan, in 1995. He has been with the National Institute of Information and Communications Technology (NICT) since October 1995, first as postdoctoral fellow and since April 1997 as permanent member of the research staff working in the fields of physical optics and optical communications.

George M. Saridis was born in Thessaloniki, Greece, in 1990. He received his Bachelor's degree from Computer Science Department, Aristotle University of Thessaloniki, where he also worked closely with photonics and optical switching for his graduation thesis. He is currently studying towards his $\mathrm{PhD}$ degree and is a member of the High Performance Networks group at University of Bristol, U.K. His research is mainly focused on optical data plane architectures for flexible, low-latency, scalable \& energy-efficient next generation optical intra Data-Centre Networks \& High Performance Computing interconnects. Among his main interests is also space division multiplexing (SDM) in conjunction with other novel technologies for future all-optical networks. His work has been accepted for oral presentation in international optical communication conferences like OFC 2015 and ECOC 2015. George is involved and has also contributed in prominent EU and UK funded research projects.

Yoshinari Awaji, biography not available at the time of publication.

Naoya Wada (M'97) received the B.E., M.E., and Dr. Eng. degrees in electronics from Hokkaido University, Sapporo, Japan, in 1991, 1993, and 1996, respectively.

In 1996, he joined the Communications Research Laboratory (CRL), Ministry of Posts and Telecommunications, Tokyo, Japan. He has been project leader of Photonic Node Project and research manager of NICT from 2006. He has been group leader of the Photonic Network Group from April 2009. Since April 2011, he has been director of the Photonic Network System Laboratory in NICT. His current research interests are in the area of photonic networks and optical communication technologies, such as optical switching network, energy-efficient network, optical access system, optical processing system, burst-mode optical communication technologies, optical packet and optical circuit integrated network, and huge capacity transmission based on spatial division multiplexing. He has published more than 100 papers in refereed English journals and more than 300 papers in refereed international conferences.

He has received the 1999 Young Engineer Award from the Institute of Electronics, Information and Communication
Engineers of Japan (IEICE), and the 2005 Young Researcher Award from the Ministry of Education, Culture, Sports, Science and Technology, Japan. He currently serves as technical program committee of many international conferences such as OFC, ECOC, OECC, ACP, and so on. He is a member of IEEE Comsoc, IEEE Photonics, IEICE, the Japan Society of Applied Physics (JSAP), and the Optical Society of Japan (OSJ). 